\title{
Device-Associated Meningitis by Linezolid-Resistant Staphylococcus haemolyticus in a Vancomycin- Hypersensitive Patient
}

\author{
Priya Vijayan ${ }^{1} \quad$ Dwarakanath Srinivas $^{2} \quad$ Nagarathna Siddaiah $^{1} \quad$ Veena Kumari Haradara Bahubali ${ }^{1}$
}

${ }^{1}$ Department of Neuromicrobiology, National Institute of Mental Health and Neurosciences, Bangalore, Karnataka, India

${ }^{2}$ Department of Neurosurgery, National Institute of Mental Health and Neurosciences, Bangalore, Karnataka, India

\begin{abstract}
Address for correspondence Veena Kumari Haradara Bahubali, MD, Department of Neuromicrobiology, National Institute of Mental Health and Neurosciences, Bangalore 560029, Karnataka, India (e-mail: docveenas2002@yahoo.com).
\end{abstract}

J Neurosci Rural Pract 2019;10:718-720

\begin{abstract}
Keywords

- postsurgical meningitis

- S. haemolyticus

- vancomycin hypersensitive

- linezolid resistance

- G2576T mutation

- cfr gene

Postsurgical device-related meningitis caused by multidrug-resistant coagulasenegative staphylococci often complicates the treatment options. We report a rare and, to our knowledge, the first clinical case report of drain-associated meningitis caused by methicillin- and linezolid-resistant Staphylococcus haemolyticus following linezolid therapy in a vancomycin-hypersensitive patient subsequently treated with cotrimoxazole, resulting in clinical improvement. The molecular mechanisms responsible for linezolid resistance were found to be the presence of G2576T mutation in domain $\mathrm{V}$ of 23srRNA region, which often arises during linezolid usage and also carriage of cfr gene, which promotes resistance independent of antibiotic pressure. We emphasize on monitoring the rational use of linezolid to avoid the spread of resistance and also comprehensive perioperative care to prevent health care-associated infection.
\end{abstract}

\section{Introduction}

Coagulase-negative staphylococci (CoNS) continue to be the predominant organism causing postsurgical drain-associated meningitis, a serious complication related to cerebrospinal

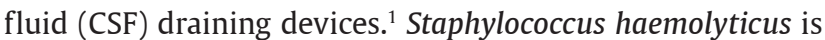
the second most frequently isolated CoNS species which displays efficient adaptability to host and hospital environment and high degree of resistance to variety of antimicrobials among all CoNS. They also act as an important reservoir for the dissemination of resistance genes to other staphylococcal species contributing to the emergence of multidrug-resistant organisms. ${ }^{2}$ Recent reports on CoNS show threatening escalation of resistance to linezolid, a potent synthetic oxazolidinone antibiotic used to treat serious gram-positive infections. ${ }^{3}$ Here, we report a rare and, to our knowledge, the first clinical case report of postsurgical drain-associated meningitis caused by methicillin- and linezolid-resistant S. haemolyticus associated with linezolid treatment in a vancomycin-hypersensitive patient.

\section{Case Presentation}

A28-year-old woman diagnosed with pituitary macroadenoma underwent endoscopic transnasal transsphenoidal decompression. Patient tolerated the surgery and postoperative computed tomography scan showed decompression of lesion. CSF rhinorrhea was noticed and a lumbar drain was placed for CSF diversion. Serial CSF samples were received for microbiological evaluation which remained sterile. A week after the surgery, the patient showed clinical symptoms such as fever, headache with drain CSF leukocyte count of 1,720 cells $/ \mathrm{mm}^{3}$ with polymorphonuclear leukocyte predominance, $176 \mathrm{mg} / \mathrm{dL}$ protein, and $1 \mathrm{mg} / \mathrm{dL}$ glucose. Adrain-associated central nervous system infection was suspected as both CSF and the drain tip yielded Methicillin-resistant CoNS (MRCoNS). Following the drain removal, the patient was started on vancomycin but developed maculopapular rash indicating drug-induced hypersensitivity reaction; hence, linezolid was started as an alternative regimen (600 mg twice daily). She received linezolid therapy for 2 weeks until CSF cultures yielded linezolid-resistant MRCoNS 
(LR-MRCoNS) which was identified as $S$. haemolyticus with minimum inhibitory concentration of linezolid being $\geq 8 \mathrm{mg} / \mathrm{L}$ by automated bacterial identification system (VITEK 2C). The antibiotic susceptibility profile showed that the isolate was sensitive to vancomycin, tetracycline, and cotrimoxazole and resistant to cefoxitin, ciprofloxacin, gentamicin, chloramphenicol, clindamycin, and rifampicin. The clinical condition of the patient improved following cotrimoxazole treatment and the isolation of LR-MRCoNS ceased in the subsequent CSFs.

Molecular studies were further undertaken to get an insight of the isolate which was confirmed as S. haemolyticus using universal primers by 16srRNA sequence analysis. ${ }^{4}$ The strain was methicillin resistant and harbored mecA gene. To understand the mechanisms of linezolid resistance, the domain $\mathrm{V}$ of $23 \mathrm{~S}$ rRNA gene was amplified, sequenced, and aligned with the corresponding nucleotide sequence of Escherichia coli (GenBank accession no V00331) and S. haemolyticus strain JCSC1435 (GenBank accession no AP006716). ${ }^{5}$ The sequencing of domain $\mathrm{V}$ of 23srRNA region revealed a G2576T (E. coli numbering) mutation. The sequence was submitted to GenBank with accession number MH156049. Furthermore, the presence of the $c f r$ gene conferring resistance to linezolid was positive by polymerase chain reaction. ${ }^{6}$

\section{Discussion}

The members of CoNS have established themselves as significant pathogens frequently seen in device-related infections. These biofilm-mediated infections are difficult to treat owing to their ability to resist host immunity as well as antibiotic penetration, often resulting in removal of the infected device. Our patient was on postsurgical external lumbar drain for the correction of CSF leakage and later developed meningitis. The major complications of such draining devices are infections resulting in meningitis or ventriculitis scaling up to $22 \%{ }^{1}$

CNS infections have limited therapeutic options as penetration of various antimicrobials through blood-brain barrier and achieving desired drug concentration in CSF is difficult. Though vancomycin is the preferred drug of choice to treat CNS infection caused by methicillin-resistant staphylococci, its poor CSF penetration has made linezolid a better choice with improved prognosis in patients. ${ }^{7}$ Linezolid-resistant Staphylococcus aureus and CoNS have been reported in separate cases and outbreaks worldwide. ${ }^{3}$ The patient had persistent CSF rhinorrhea postoperatively which warranted insertion of external lumbar drain. Risk of drain-associated infections occurring at the time of insertion or during routine care and manipulation is common. In addition, the transnasal-transsphenoidal surgical approach, involving the passage through the nasal cavity which harbors several normal flora also would have contributed to meningitis.

During the course in the hospital, patient had a preliminary MRCoNS lumbar drain infection by a linezolid susceptible strain, but as on linezolid therapy, the strain acquired resistance which is a common feature. ${ }^{3}$ Mutation, recombination events happening during the treatment or with prior antibiotic exposure, is responsible for resistance. Acquisition of $c f r$ gene even in the absence of antibiotic pressure because of the low fitness cost in maintaining the gene also contributes to resistance. ${ }^{8}$ Our isolate harbored both the aforementioned mechanisms of linezolid resistance. It possessed the G2576T mutation in domain V of 23srRNA region, the most frequently reported mutation found in many staphylococcal and enterococcal isolates, linked with exposure to linezolid. ${ }^{9,10}$ Our isolate also harbored the $c f r$ gene, a highly effective plasmid-mediated gene known to impart resistance independent of linezolid usage, which easily gets disseminated through horizontal transfer thus spreading resistance. These isolates may act as a reservoir for resistance which is worrisome in a hospital setup.

In our patient, though the isolate was vancomycin susceptible, it was discontinued due to hypersensitive reaction. Linezolid, the next choice drug was initiated to which resistance developed subsequently, thus drastically limiting the treatment options. As a result, cotrimoxazole, a broadspectrum antibiotic with good central nervous system penetration was started, to which the patient responded. Cotrimoxazole in the treatment of meningitis caused by linezolid-resistant S. epidermidis and an unusual CoNS, S. chromogenes has been previously reported. ${ }^{11,12}$

With enormous increase in the use of indwelling devices and device-related infections caused by resistant isolates, our report warrants comprehensive perioperative care taking into account the risk factors, to significantly reduce the infection and improve patient care.

\section{Conclusion}

Linezolid continues to be the preferred alternative drug for CNS infections caused by methicillin-resistant staphylococci due to its good CSF penetration, effectiveness, few side effects, and low cost. Clinicians should be aware that linezolid resistance may develop any time during the treatment; hence, continuous monitoring is essential for timely identification of resistance. Strict adherence to rational infection control practices and judicious use of linezolid are highly recommended to prevent the spread of resistance and preserve the drug.

\section{Funding}

None.

\section{Conflict of Interest}

None declared.

\section{References}

1 Soavi L, Rosina M, Stefini R, et al. Post-neurosurgical meningitis: management of cerebrospinal fluid drainage catheters influences the evolution of infection. Surg Neurol Int 2016;7(Suppl 39):S927-S934

2 Czekaj T, Ciszewski M, Szewczyk EM. Staphylococcus haemolyticus - an emerging threat in the twilight of the antibiotics age. Microbiology 2015;161(11):2061-2068

3 Gu B, Kelesidis T, Tsiodras S, Hindler J, Humphries RM. The emerging problem of linezolid-resistant Staphylococcus. J Antimicrob Chemother 2013;68(1):4-11 
4 Lane D, 16S/23S rRNA sequencing. In: Stackebrandt E, Goodfellow M, eds. Nucleic Acid Techniques in Bacterial Systematics. New York: John Wiley; 1991 115-175

5 Meka VG, Pillai SK, Sakoulas G, et al. Linezolid resistance in sequential Staphylococcus aureus isolates associated with a T2500A mutation in the 23S rRNA gene and loss of a single copy of rRNA. J Infect Dis 2004;190(2):311-317

6 Kehrenberg C, Schwarz S. Distribution of florfenicol resistance genes fexA and cfr among chloramphenicol-resistant Staphylococcus isolates. Antimicrob Agents Chemother 2006;50(4):1156-1163

7 Sipahi OR, Bardak-Ozcem S, Turhan T, et al. Vancomycin versus linezolid in the treatment of methicillin-resistant. Staphylococcus aureus. meningitis. Surg Infect (Larchmt) 2013;14(4):357-362

8 LaMarre JM, Locke JB, Shaw KJ, Mankin AS. Low fitness cost of the multidrug resistance gene cfr. Antimicrob Agents Chemother 2011;55(8):3714-3719
9 Hill RL, Kearns AM, Nash J, et al. Linezolid-resistant ST36 methicillin-resistant Staphylococcus aureus associated with prolonged linezolid treatment in two paediatric cystic fibrosis patients. J Antimicrob Chemother 2010;65(3):442-445

10 Rai S, Niranjan DK, Kaur T, Singh NP, Hada V, Kaur IR. Detection of the classical G2576U mutation in linezolid resistant Staphylococcus aureus along with isolation of linezolid resistant Enterococcus faecium from a patient on short-term linezolid therapy: first report from India. Indian J Med Microbiol 2015;33(1):21-24

11 Vena A, Falcone M, Comandini E, et al. Daptomycin plus trimethoprim/sulfamethoxazole combination therapy in post-neurosurgical meningitis caused by linezolid-resistant Staphylococcus epidermidis. Diagn Microbiol Infect Dis 2013;76(1):99-102

12 Batra P, Deo V, Mathur P, Gupta AK. Cotrimoxazole, a wonder drug in the era of multiresistance: case report and review of literature. J Lab Physicians 2017;9(3):210-213 